\title{
Tratamiento con Tiopental del Estado Epiléptico en Niños
}

\author{
Dr. Mario Cerda S.1, Dr. Juan C. Casterán V.1. Dr. Rolando Sạavedra O.l, \\ Srta. Cecilia Cofré L. ${ }^{2}$, Srta. Marisa Del Valle M. ${ }^{2}$, Srta. Sara Peña R. ${ }^{3}$ \\ Treatment of Status Epilepticus in Children
}

\begin{abstract}
Ten children admitted to the Pediatric intensive care unit of Sótero del Rio Hospital were included in this protocol, all of the, with Status Epilepticus secondary to different C.N.S. diseases and previously managed in the wsual way without imporvement. Sodium Thiopental in increasing doses by continous I.V. infusion was then started and all patients stoped convulsing. The mean treatment period was 24 hrs., with a wide range in medication doses.

Method of administration, monitoring and signs of overdose, are described and a treatment schedule is proposed.
\end{abstract}

El Síndrome convulsivo secundario se presenta frecuentemente en las enfermedades del S.N.C., del nin̄o, agregando a ellas un factor de agravamiento de las condiciones generales y siendo no pocas veces causa de muerte "per se". Generalmente los factores causales de esta condición son múltiples (edema, infección, hipoxia, hipoglicemia, acidosis, alteraciones electroliticas), muchas de ellas son a su vez consecuencia de la convulsión prolongada, determinando un círculo vicioso que deberá cortarse precozmente si se quiere influir en el pronóstico del paciente.

Por otra parte la administración excesiva de anticonvulsivantes parenterales (Fenobarbital, Diazepám) solos 0 asociados agregan depresión respiratoria como nuevo riesgo cuando no han controlado la convulsión.

Habiendo usado en varias ocasiones el Tiopental sódico en infusión E.V., continua, nos llamó la atención la falta de datos, respecto a dosis, técnica de administración y monitoreo de parámetros vitales para su manejo seguro.

Esto nos ha llevado a diseñat un esquema de tratamiento y manejo de esta condición, que intentamos evaluar.

\section{MATERIAL Y METODO}

Ingresamos a este estudio prospectivo aquellos pacientes que presentaron an síndrome convulsivo generalizado, mantenido o reiterado, no controlable con la terapia disponible habitualmente: fenobarbital, diazepám ev., clonazepam oral o ev., difenil hidantoina oral, ev., etc. (Estado Convalsiwo); hospitalizados en la Unidad de Cuidados

1 Médicos UCI. Pediátrica Hospital D. Sótero del RíoDpto. Pediatr ja Universidad Católica de Chile.

2 Inter nas de Medicina Universidad Católica de Chile.

3 Enfermera UCI Pediátrica Hospital Dr. Sótero del Río.
Intensivos del Servicio de Pediatría, Hospital Sótero del Río.(U.C.I.P.), en el período comprendido entre Enero de 1978 a Enero de 1982. Tomada la decisión de instalar este tratamiento, se conectó el paciente a ventilador mecánico, (requjsito indispensable en esta técnica), con parámetros variables según edad mediante intubación nasotraqueal. Se realizó contral continuo del Electrocardiograma en monitor y registro horario de frecuencia respiratoria, temperatura axilar y presión arterial con manguito (en 1 paciente se controló presión arterial media con catéter intra-arterial y transductor de presión).

E1 Tiopental sódico se administró por vía venosa en una solución glucosada al $5 \%$, en un bolo inicial de $2-4 \mathrm{mg} / \mathrm{Kg}$., para continuar con infusión continua con bomba en dosis crecientes de 2--8 $\mathrm{mg} / \mathrm{Kg}$, por hora (ent un caso por mal cálculo de dosis se administró $9 \mathrm{mg} / \mathrm{Kg}$ hora). Al alcanzar la dosis útil (dosis sin convulsión) esta se mantuvo durante 24 horas.

Antes de suspenderlo, se administró nuevamente anticonvulsivantes de mantención (fenobarbital en dos de $5-7 \mathrm{mg} / \mathrm{Kg} /$ día).

La infusión de Tiopental se mantuvo por periodos de 1 hora, antes de juzgar que no habia respuesta, lo cual se determinaba por persistencia de actividad convulsiva de cualquier tipo.

Otros parámetros que se vigilaron fueron tonicidad inuscular, reflejos osteotendíneos, tamaño de pupilas y reacción foto-motora.

Se interpretó como sobredosis cuando aparecía hipotonía, hiporreflexia y miosis pupilar con escasa respuesta a la luz; y como intoxicación cuando aparecieron signos de depresión vasomotora: tendencia a la bradicardia, hipotensión y enfriamiento.

En estos casos se suspendió la infusión o se disminuyó a la mitad con mejor ía de estos signos a los pocos minutos y se pasó a terapia de mantención. 


\section{Caso Indice}

L.E.F., Preescolar de 2 años y 7 meses, sin antecedentes perinatales ni mórbidos de importancia. Siete dias antes del ingreso presenta bruscamente fiebre $39-40^{\circ} \mathrm{C}$ con decaimiento y odinofagía. Concurre a un Servicio de Urgencia diagnosticándose amigdalitis aguda y tratándosele con Penicilina Benzatina y Aspirina. Persiste decaído durante los sigujentes cuatro días, apareciendo somnolencia y alteraciones conductuales, indiferencia al medio y sonrisa sin motivo.

El día previo a su ingreso presenta cuadro convulsivo tónico-clónico generalizado de cinco a diez minutos de duración que es tratado en un Servicio de Urgencia con Diazepam ev., practicándole Punción lumbar (P.L.), cuyo Liquido céfaloraquídeo (L.C.R.), resulta claro y transparente, desconociéndose el estudio citoquímico. De vuelta a su casa además de la somnolencia lo notan muy agitado y agresivo; al día siguiente es traído al Servicio de Urgencia de nuestro Hospital convulsionando. Se le admínistra Diazepám e.Y., $10 \mathrm{mg}$. y $50 \mathrm{mg}$., de Fenobarbital i.m.

Al ingreso se aprecia soporoso, con períodos de excitación hipotonia e hiporreflexia osteotendínea simétrica, respuesta plantar de extensión bilateral, con fondo de ojo normal y sin signos de irritación meníngea.

El L.C.R., se aprecia claro y transparente con 10 leucocitos por $\mathrm{mm}^{3}$ como única alteración; el resto de los exámenes; glicemia, ionograma, hemograma, eritrosedimentación y transaminasas normales.

Se deja con fenobarbital $10 \mathrm{mg} / \mathrm{Kg}$., IM., cada 12 horas y ampicilina $200 \mathrm{mg}$., por presentar signos de congestión timpánica bilateralmente.

Durante los tres primeros días persiste con febrículas, períodos de apatia, alternados con excitación y verborrea.

Al tercer dia presenta nuevamente convulsiones que ceden con Diazepam Ev., en bolo, se agrega clonazepam $0,25 \mathrm{mg} / \mathrm{Kg} /$ día. Pese a esto el paciente persiste con alteraciones conductuales y de conciencia; sub-febril y con convulsiones intermitentes. El L.C.R., revela elevación de las proteínas de hasta $140 \mathrm{mg} . \%$ con resto de valores normales. Los otros valores biométricos sanguíneos no se alteran. El ecoencefalograma es normal y el E.E.G., revela signos de hiper excitabilidad neuronal difusa, simétrica.

Al $11^{\circ}$ dia de hospitalización, por presentar convulsiones subintrantes, comprometiendo las funciones vitales, se trasladó a U.C.I.P. en donde se decide tratar con Tiopental en infusión Ev., continua. Previa intubación nasotraqueal se conecta a respirador Bird Mark-8, con ventilación controlada manteniéndose $\mathrm{PO}_{3}$ entre 100 y $120 \mathrm{mmHg}$ y $\mathrm{PCO}_{2}$ entre 25 y $30 \mathrm{~mm} \mathrm{Hg}$.

Se controla frecuencia cardíaca con monitor cardíaco y presión arterial cada hora.

Se administra Tiopental Sódico en bolo de $3,5 \mathrm{mg} \times \mathrm{Kg}$., Ev., continuándose con infusión continua de $3 \mathrm{mg} / \mathrm{kg} / \mathrm{hora}$, con lo cual ceden transitoriamente, reapareciendo posteriormente por lo que se debe aumentar sucesivamente a 4-6 y 8 $\mathrm{mg} / \mathrm{kg} /$ hora por persistir con actividad convulsiva. Se mantiene sin convulsionar por 24 horas con la última dosis. Sin embargo al cabo de este período, se detecta Hipotensión, Bradicardia e Hipotermia, por lo que se suspende el tratamiento continuándose con fenobarbital $7 \mathrm{mg} / \mathrm{kg} / \mathrm{IM}$., al dia.

Se mantiene con fenobarbital y clonazepan sin volver a presentar convulsiones durante los próximos 15 días de hospitalización, en este periodo su nivel de conciencia mejora gradualmente, conectấndose progresivamente con el ambiente.

E) L.C.R., previo al alta es normal y el E.E.G., muestra signos de bajo umbral convulsivo sin signos focales.

Se da de alta en buenas condiciones generales y de conciencia, sin déficit neurológicos con los diagnósticos de:

\section{Eutrofia}

2. Encefalitis viral

3. Sindrome convulsivo secundario

Se controla en Policlinico de Neurología en donde a raíz de suspensión del tratamiento presenta convulsiones ocasionales, lo cual se corrige exitosamente estando 6 meses sin recurrir manteniéndose en tratamiento con ácido valproico.

\section{RESULTADOS (Tabla 1)}

Nuestros pacientes se distribuyeron en forma dispersa a través de todo el período de la infancia, desde $\operatorname{los} 20$ días hasta los 14 años. De los pacientes 3 fueron del sexo femenino y 7 del masculino, el estado nutritivo era satisfactorio en todos ellos.

En cuanto a los diagnósticos de base 6 presentaban una meningitis aguda (M.B.A.), dos presentaban la complicación convulsiva al ser tratados con Metotrexato intratecal por leucernia linfoblástica Aguda (L.L.A.), uno presentaba sepsis por Klebsiella y encefalopatía tóxico-metabólica y uno encefalitis viral.

En 5 de ellos se pesquisó clínicamente la presencia de edema cerebral agudo y en el resto no había signos evidentes de tal complicación, sin embargo en todos con excepción de un paciente 
Tabla 1.

\begin{tabular}{|c|c|c|c|c|c|c|c|c|c|c|}
\hline PAC. NO & 1 & 2 & 3 & 4 & $s$ & 6 & 7 & 8 & 9 & 10 \\
\hline EDAD & Im. $7 \mathrm{ds}$ & $14 \mathrm{a}$ & $20 \mathrm{ds}$ & $\operatorname{lm} .15 \mathrm{ds}$ & 41/2 a. & 2a. $7 \pi$ & $2 \mathrm{~ms}$ & I1 $\mathrm{ms}$. & $4 \mathrm{~ms}$. & 12 вйо \\
\hline Diag. Principal & $\begin{array}{l}\text { - Sopsis a } \\
\text { Klebsielia } \\
\text { - Encefalopa. } \\
\text { lía tókica }\end{array}$ & L.L.A. & M.B.A. & M.B.A. & L.L.A. & $\begin{array}{l}\text { Enceraii- } \\
\text { lis ag. }\end{array}$ & M.E.A. & M.B.A. & M.B.A. & M.B.A. \\
\hline $\begin{array}{l}\text { Ederma } \\
\text { Cerebral clínico }\end{array}$ & - & - & + & $t$ & - & - & + & + & + & - \\
\hline $\begin{array}{l}\text { Periodo Corvulsivo } \\
\text { previo al trat. } \\
\text { con Tiopea 1al }\end{array}$ & 4 dias & 3 hrs. & 2 dias & $11 / 2 \mathrm{ds}$ & 1 hora & 11 días & 2 dias & 48 hrs. & 4 hrs. & 3 hrs. \\
\hline $\begin{array}{l}\text { Dosis de carga } \\
(\mathrm{mg} / \mathrm{Kg})\end{array}$ & 5 & 6 & 4 & - & 6 & 3.5 & 1 & 4 & 2 & 2 \\
\hline $\begin{array}{l}\text { Dosis de manter- } \\
\text { ción }(\mathrm{mg} / \mathrm{Kg} / \mathrm{hr} \text {.) }\end{array}$ & 3 & 2 & 2 & 8 & $B$ & 3 & 1 & 6 & I & 9 \\
\hline $\begin{array}{l}\text { Dosis de Recurren- } \\
\text { cia, (mg/K//hI) }\end{array}$ & - & 2 & 6 & 8 & - & 4 & 3 & - & 3 & - \\
\hline $\begin{array}{l}\text { Dosis sin convul- } \\
\text { sion (mg/Kg/hr.) }\end{array}$ & 3 & B & 46 & $* 8$ & 8 & 8 & 6 & 2 & $\bullet 8$ & g \\
\hline $\begin{array}{l}\text { Signos Intoxi- } \\
\text { cación. F.C. }\end{array}$ & - & $\downarrow$ & - & $\downarrow$ & - & $t$ & - & - & - & - \\
\hline P.A. & - & $\downarrow$ & . & $t$ & - & $\downarrow$ & - & - & - & - \\
\hline$T^{0}$ & - & $\downarrow$ & - & 4 & - & $\downarrow$ & - & $\cdot$ & - & - \\
\hline Dosis & - & 8 & - & 8 & - & 8 & - & - & - & - \\
\hline $\begin{array}{l}\text { Dosis Miosis } \\
\text { (mg/Kg/hr.) }\end{array}$ & - & - & 6 & - & 6 & 4 & 6 & 6 & - & - \\
\hline $\begin{array}{l}\text { Dosis Alt. Reflejo } \\
\text { y lono (mg/Kg/hr.) }\end{array}$ & 4 & - & - & 6 & 6 & 6 & 6 & 4 & - & 6 \\
\hline \multirow[t]{2}{*}{$\begin{array}{l}\text { Trat. Anti-edems } \\
\text { cerebral }\end{array}$} & + & + & + & + & - & + & + & + & + & + \\
\hline & & & $\begin{array}{l}\text { *bolo de } \\
4 \mathrm{mg} / \mathrm{K}_{\mathrm{g}}\end{array}$ & $\begin{array}{l}\text { "bok ds } \\
6 \mathrm{ng}, \mathrm{K}_{\mathrm{B}}\end{array}$ & & & & & $\begin{array}{l}\text { *bolo de } \\
3 \mathrm{mg} / \mathrm{Kg}\end{array}$ & \\
\hline
\end{tabular}

(No 5) se efectuó tratamiento de edema cerebral con manitol, corticoides, restricción de liquidos e hiperventilación. En el paciente en que no se usó, no se juzgó oportuno por la corta evolución del estado convulsivo previo al tratamiento con Tiopental.

Todos los pacientes recibieron tratamiento de su enfermedad de base (Antibióticos y Antileucémicos).

E1 periodo de convulsiones previo al tratamiento con tiopental fluctuó entre 1 hora y 48 horas, con excepción de un paciente que es el que se rełata en que fue de 11 dias. Durante este período se usó anticonvulsivantes tradicionales en dosis juzgadas como suficientes. Todos recibieron Fenobarbital y Diazepam en dosis repetidas y estimadas como útiles excepto 3 pacientes (uno de ellos recibió Clonazepam y Fenobarbital, en otro se agregó al Fenobarbital Clonazepam y otro recibió Fenobarbital y Fenitoína E.v.).

Sólo 2 pacientes permanecieron convulsionando un período limitado de tiempo, 1 y 3 horas respectivamente. Ambos portadores de L.L.A., en tratamiento con Metotrexato intratecal, complicación que se atribuyó al diluyente. Por ser pacientes sin daño neurológico previo con convulsiones reiteradas se inició precozmente el procedimiento.

El tiempo de tratamiento con Tiopental fluctuó entre 16 y 90 horas.

La dosis útil, es decir la dosis en la cual desaparecieron las convulsiones, fluctuó entre 2 y 9 $\mathrm{mg} / \mathrm{kg} / \mathrm{hora}$ ( 5 pacientes con $8 \mathrm{mg} / \mathrm{kg} / \mathrm{hora}, 2$ con $6 \mathrm{mg} / \mathrm{kg} / \mathrm{hora}, 2 \operatorname{con} 3$ y $2 \mathrm{mg} / \mathrm{kg} /$ hora respectivamente y 1 con $9 \mathrm{mg} / \mathrm{kg} /$ hora).

Hubo recurrencia en 6 pacientes, pero en sólo dos de ellos la recurrencia apareció con dosis altas (6-8 mg/ $/ \mathrm{kg} /$ hora), esto obligó al uso de un bolo adicional presentando uno de ellos síntomas de intoxicación por lo que se debió disminuir la dosis a la mitad. En los otros 4 pacientes que presentaron recurrencia convulsiva con dosis que fluctuaban entre 2 y $4 \mathrm{mg} / \mathrm{kg} /$ hora y se logró coartar el 
cuadro aumentando las dosis de infusión entre $5 \mathrm{y}$ $8 \mathrm{mg} / \mathrm{kg} /$ hora y adicionando un bolo de $2 \mathrm{mg} / \mathrm{kg}$., en uno de ellos.

Otros 2 pacientes en que se usó 8 y 9 $\mathrm{mg} / \mathrm{kg} /$ hora presentaron tambiên signos de sobre dosis (miosis, hipotonia y arreflexia) por lo que se debió bajar la dosis no recurriendo en ninguno de ellos las crisis convulsivas durante su hospitalización.

En 5 pacientes se pesquisó miosis en niveles de administración en 4-6 $\mathrm{mg} / \mathrm{kg} / \mathrm{hora}$.

El tono muscular y los reflejos osteotendínicos se deprimieron siempre sobre $\operatorname{los} 4 \mathrm{mg} / \mathrm{kg} /$ hora, presentando esta alteración, 7 de nuestros pacientes. En los casos en que por efecto de las dosis elevadas se produjo hipotensión, estas no fueron importantes y cedieron rápidamente al disminuir las dosis o suspender el medicamento. En ningún caso se juzgó necesario utilizar vasopresores.

Con esta terapia todos los pacientes dejaron de convulsionar no reincidiendo una vez suspendida, un paciente falleció a causa de su M.B.A., lo que ocurrió varias horas después de suspendido el tratamiento y sin haber vuelto a convulsionar.

\section{DISCUSION}

El Tiopental sódico, es un derivado del ácido barbitúrico de acción ultra breve debido a su alta liposolubilidad, propiedad que le confiere disminución en la duración de su acción y de la latencia hasta el comienzo de su actividad.

Penetra rápidamente las membranas una vez administrado, siendo captado eficazmente por áreas más vascularizadas del encéfalo, órgano exquisitamente sensible. La captación máxima se produce a los 30 segundos.

Su acción puede resumirse en "Depresión de los tejidos excitables", inhibiendo transitoriamente las respuestas monosinápticas, actuando sobre la liberación de neurotrasmisores en terminales presinápticos.

Su afinidad por la masa encefálica sumado a la breve latencia y a la acción depresiva lo hacen de gran utilidad en el manejo de las crisis convulsivas $^{1-2-3-4 \cdot 5}$

Cuando alcanza niveles elevados en el plasma se producen efectos derivados de su actividad depresora sobre otras áreas produciéndose disminución de los reflejos inicialmente superficiales y más tarde profundos, las pupilas se tornan mióticas, cae la presión arterial y disminuye el gasto cardiaco ${ }^{6}$.

En nuestra serie el estado convulsivo se presentó más frecuentemente en paciertes con meningitis bacteriana aguda ( 6 casos) lo que se explica por la alta incidencia de ta complicación convulsiva en dicha enfermedad ${ }^{7}$.
El edema cerebral es un factor agregado frecuente en el Estado convulsivo, encontrándolo.en 5 de nuestros pacientes, el tratamiento antiedema lo justificamos por lo anterior y por el hecho de la poca certeza clínica como diagnóstico exclusivo de ederna ${ }^{8}$.

Nos parece evidente que todo paciente que evoluciona con un estado convulsivo prolongado sólo podrá ser tratado en una U.C.I.P., ya que la disponibilidad y complejidad de las técnicas a usar junto con el monitoreo continuo sólo puede ser efectuado en estos sitios.

Del tratamiento realizado en nuestros pacientes se concluye que la dosis de carga inicial de Tiopental sódico parece indispensable ya que en el único caso que no se usó (paciente $\mathrm{N}^{\circ} 4$ ), debió administrarse con posterioridad ante la recurrencia de convulsiones.

Sugerimos una dosis de carga inicial de $2-4$ $\mathrm{mg} / \mathrm{kg} / \mathrm{Ev}$, en un período de 2 a 3 minutos, fundamentado en el hecho de que dosis mayores produjeron signos precoces de sobre dosis y no impidieron la recurrencia que fue más dependiente de las dosis de mantención.

Las dosis elevadas de $6-8 \mathrm{mg} / \mathrm{kg} /$ hora recomendamos usarlas sólo hasta coartar la convulsión. Una vez obtenido este resultado durante más de $\mathbf{3 0}$ minutos, debiera disminuirse a dosis menores, (4-5 mg/kg/hora) ya que si bien por períodos cortos estas dosis no son peligrosas (incluso en miocardio dañados disminuyen el consumo de oxígeno, sin disminuir el gasto cardiaco) 9, en infusiones continuas y prolongadas la eliminación de la droga disminuye produciéndose acumulación de la misma, lo que no sucede a dosis inferiores de $4-5 \mathrm{mg} / \mathrm{kg} /$ hora $(10-11)$. Estas últimas serían las dosis recomendadas para mantención.

Esto lo constatamos clínicamente, en nuestros pacientes, sin embargo, las, complicaciones serias (Hipotensión, Bradicardia e Hipotermia) fueron graduales, fácilmente detectables y no requirieron tratamiento, al suspender o disminuir significativamente la droga.

En todos nuestros pacientes el buen resultado se obtuvo en periodos que fluctuaban entre $16 \mathrm{y}$ 90 horas, con un promedio de 24 horas.

El tratamiento debe ser efectuado durante el tiempo mínimo necesario para coartar el sindrome, asi evitar complicaciones inherentes a técnicas empleadas como por ejemplo el uso de ventilación mecánica por períodos prolongados.

El éxito de este esquema puede atribuirse en parte a la acción anticonvulsivante del Tiopental pero también al efecto anti-edema cerebral del mismol 2-13-14-15 siendo un esquema terapéutico capaz de abolir el estado convulsivo en forma rápida, eficaz y sin complicaciones sí, su uso está 
supeditado a un riguroso monitoreo y contral, teniendo en mente los signos derivados de la sobre dosis que preceden a la intoxicación.

Una desventaja del procedimiento es que debe utilizarse obligatoriamente con ventilación mecánica, lo que lo hace limitado a Unidades más especializadas y usado sólo en aquellos pacientes en que los tratamientos habituales en dosis máximas han resultado inefectivos.

Es evidente que un monitoreo, instantáneo y seriado de los niveles sangúneos del barbitúrico eliminaría aún más los riesgos del procedimiento, pero esta es una posibilidad con lo cual no contamos habitualmente en nuestro medio.

\section{RESUMEN}

Se estudió el efecto del Tiopental sódico en el manejo del Estado Convulsivo en 10 pacientes hospitalizados en U.C.I.P., del Hospital Dr. Sótero del Rio.

Se analizan en detalle las dosis usadas y los efectos de sobre-dosís del fârmaco, proponiéndose un esquema terapéutico útil para coartar el Síndrome convulsivo y evitar efectos colaterales.

Se describen las técnicas usadas y los resultados de este Síndrome.

Se concluye que este esquema terapéutico es de gran utilidad en el estado convulsivo logrando remision de las crisis convidsivas en todos los pacientes, siempre que se utilice en las dosis adecuadas y con un cuidadoso morlitoreo de los signos vitales.

\section{REFERENCIAS}

1 Brown As., Horton J.M. "Status epllepticus treated by intravenous infusion of Thiopentone sodium. Brit. Med. Journal; 1: 27, 1967.

2 Dundes J.W., Grey R.C. "Thipentone in status epileticus". Brit. Med. Journal; 1: 362, 1967.

3 Henliwel $M$. , Flonggan $R . J$. "Thipentone infusions" Anaesthesia; 35: 78-79, 1980.

4 Feneck-R.O.: "A case of status epilepticus", Anaesthesia; 36: 691-695, 1981.

5 Levin D., Morris F., Moore G., "A Practical Pediatric guide to Pediatric Intensive care" St. Louis W.B. Saunders Co. 9: 36-38, 1979.

"Goodman and Gilman: "Hypnotics and Sedatives" Chapter 9, p. 102: Fith Edition 1975. The Mac Millan Co., td. New York.

${ }^{7}$ Feigin R.O. Dooge P.R.: Bacterial Meningitis: Newer concepts of Pathophysiology and Neurologic sequelae". Ped. Clin. N.A. 23: 541, 1976.

"Eishman, R.: "Brain Edema". New. Eng. J. Med. 2: $706,1975$.

${ }^{9}$ Reiz S., Balfors E., Frtedman A., Hazgmark S., Peter $T$. "Effects of Thiopentone on cardiac Perfomance, Coronary Hemodynamies and miocardial Oxygen consumption in chronic Ischemic Heart Disease. "Acta anaesth. Scand. 25: $103-110,1981$.

10 Mihm F.G., Stanski D.R., Rosenthal M.H.: "Zeto order pharmacokinetics of high dose Thiopental". Anesthesiology 51: 204, 1979.

11 Stawksi D.R. "Pharmacokinetics modelling of Thiopental". Anesthesiology 54: 446-448, 1981.

12 Batzdorf: "The management of cerebral edema in pediatric practice". Pediatrics 58: 78, 1976.

13 Breinic and col.: "Clinical featibility triales of barbiture therapy". Crit. Care Med. 6:228, 1978.

14 Cohn M.L. "Penthobarbithal alteration of AMPC Metabolism". Anesthesiol. 51: 545, 1979.

is Grenvik A., Safar P., "Clinics in critical care medicine: Brain Failure and resuscitation" Churchill Livingstone Ed., NY. 231, 1981. 